\title{
On n-Weakly Regular Rings
}

\section{Raida D. Mahammod}

College of Computer Sciences and Mathematics

University of Mosul

Received on: 09/05/2010

\author{
Mohammed Th. Al-Neimi \\ College of Engineering \\ University of Mosul
}

Accepted on: 29/06/2010

\begin{abstract}
As a generalization of right weakly regular rings, we introduce the notion of right $n$-weakly regular rings, i.e. for all $a \in N(R), a \in a R a R$. In this paper, first give various properties of right $n$-weakly regular rings. Also, we study the relation between such rings and reduced rings by adding some types of rings, such as NCI, MC2 and SNF rings.
\end{abstract}

Keywords: Weakly Regular Rings, Reduced Rings.

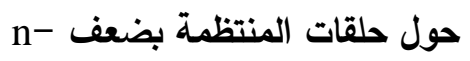

محمد ذنون آل نعمة

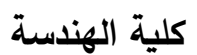

جامعة الموصل
تاريخ القبول: 2010/06/29

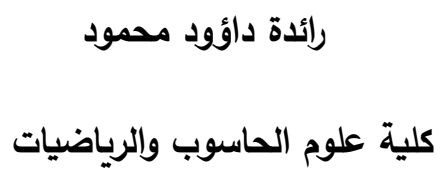

تاريخ الاستلام: 2010/05/09

\footnotetext{
الملخص

كتعميم للحلقـات المنتظمـة بضـفف، نُعَرِّف الحلقـات المنتظمـة بضـفـف مـن النمط n على إنها لكل

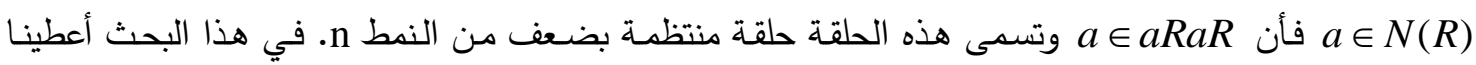

خواص متتوعة للحلقات المنتظمة بضعف من النمط n وكذلك درسنا العلاقة بين تلك الحلقات والحلقات المختزلة فئل

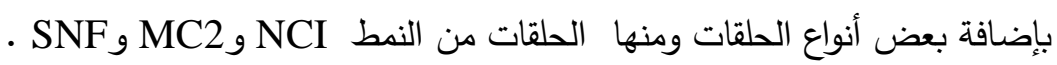

الكلمات المفتاحية : الحلقات المنتظمة بضعف، الحلقات المختزلة.
}

\section{.Introduction:}

Throughout this paper a ring $R$ denotes an associative ring with identity and all modules are unitary. For a subset $X$ of $\mathrm{R}$, the left(right) annihilator of $X$ in $R$ is denoted by $r(X)(l(X))$. If $X=\{a\}$, we usually abbreviate it to $r(a)(l(a))$. We write $J(R)$, and $N(R)$, for the Jacobson radical and the set of nilpotent elements respectively.

The center of the ring $\mathrm{R}$ is denoted by $\operatorname{Cent}(R)$ and it is $\operatorname{Cent}(R)=\{a \in R / a r=r a \quad \forall r \in R\}$. A ring $\mathrm{R}$ is called n-regular if for all $\mathrm{a} \in N(R)$, $\mathrm{a} \in \mathrm{aRa}$ [7]. A right $R$-module $M$ is called $N$ flat if for any $a \in N(R)$, the mapping $1_{\mathrm{M}} \otimes \mathrm{i}: \mathrm{M} \otimes{ }_{\mathrm{R}} \mathrm{Ra} \rightarrow \mathrm{M} \otimes \mathrm{R}_{\mathrm{R}} \mathrm{R}$ is monic, where $i: R a \rightarrow R$ is the inclusion mapping [8]. A ring $\mathrm{R}$ is called right (left) SNF if every simple right(left) R-module is $N$ flat [8]. 
A ring $\mathrm{R}$ is called semiprime if it has no nilpotent ideals [6]. A ring $\mathrm{R}$ is called reduced if $N(R)=0$ [6], or equivalently, $a^{2}=0$ implies $a=0$ in $\mathrm{R}$ for all $a \in R$. Recall that a ring $\mathrm{R}$ is $M E R T$ (resp. MELT), if every maximal essential right (resp. left) ideal of $\mathrm{R}$ is an ideal [9].

\section{2. n - Weakly Regular Ring}

This section is devoted to give the definition of n-weakly regular rings with some of its characterizations and basic properties.

A ring $\mathrm{R}$ is right (left) weakly regular [6], if $\mathrm{a} \in \mathrm{aRaR}(\mathrm{RaRa})$ for every $\mathrm{a} \in \mathrm{R}$. We called $\mathrm{R}$ is weakly regular if it is both right and left weakly regular.

\section{Definition 2.1}

A ring $\mathrm{R}$ is to be right (left) $n$-weakly regular if $\mathrm{a} \in \mathrm{aRaR}(\mathrm{a} \in \mathrm{RaRa})$ for all $a \in N(R)$. We say that $R$ is n-weakly regular if it is right and left n-weakly regular ring.

Clearly every weakly regular rings are $n$-weakly regular.

\section{Examples:}

1- Every reduced ring is n-weakly regular.

2- Every n-regular ring is n-weakly regular ring.

3- The ring $Z_{6}$ of integers modulo 6 , is reduced, n-regular, weakly regular ring, so it is n- weakly regular.

4- Let $R=\left\{\left[\begin{array}{ll}Z_{2} & Z_{2} \\ Z_{2} & Z_{2}\end{array}\right]\right\}, N(R)=\left\{\left[\begin{array}{ll}0 & 0 \\ 0 & 0\end{array}\right],\left[\begin{array}{ll}0 & 1 \\ 0 & 0\end{array}\right],\left[\begin{array}{ll}0 & 0 \\ 1 & 0\end{array}\right],\left[\begin{array}{ll}1 & 1 \\ 1 & 1\end{array}\right]\right\}$. $R$ is $\quad \mathrm{n}$ regular, weakly regular ring, so it is $\mathrm{n}$ - weakly regular but $\mathrm{R}$ not reduced ring.

5- The ring $Z$ of integer number is reduced, n-regular, so it is n- weakly regular but $\mathrm{Z}$ is not weakly regular ring.

\section{Proposition 2.2} all $a \in N(R)$.

$\mathrm{R}$ is a right $\mathrm{n}$-weakly regular ring if and only if a $\mathrm{R}$ is idempotent right ideal for Proof:

Let $\mathrm{R}$ is a right $\mathrm{n}$-weakly regular ring and $\mathrm{I}$ is a principal right ideal of $\mathrm{R}$ generated by a nilpotent element, then there exists $a \in N(R)$ such that $I=a R$, clearly $I^{2} \subseteq I$.

On the other hand, since $\mathrm{R}$ is right $\mathrm{n}$-weakly, then there exists $\mathrm{y}, \mathrm{z} \in \mathrm{R}$ such that $a=a y a z$. Now let $x \in I$, then there exists $r \in R$ such that $x=a r=a y a z r \in I^{2}$. Therefore $I \subseteq I^{2}$. Hence $I^{2}=I$.

Conversly, Let $a \in \mathrm{N}(\mathrm{R})$, since $a \mathrm{R}$ is idempotent right ideal of $\mathrm{R}$, so $\mathrm{a} \in \mathrm{aR}=\mathrm{aRaR}$. Therefore $\mathrm{R}$ is right $\mathrm{n}$-weakly regular ring.

\section{Proposition 2.3}

Let $R$ be a right $n$-weakly regular ring. If $a R \subseteq I$, for $a \in N(R)$ and $I$ is right or left ideal. Then $\mathrm{aRI}=\mathrm{aR}$.

Proof:

It is clearly that $b R I \subseteq b R$ for any $b \in R$. Now let $a \in N(R)$ and $x \in a R$, then there exists $r \in R$ such that $x=a r$. Since $R$ is right $n$-weakly regular ring then there exists $y, z \in R$ such that $a=a y a z, x=a y a z r$, hence $a z r \in a R \subseteq I$. So $a R \subseteq a R I$. Therefore $a R I=a R$. 


\section{Corollary 2.4}

Let $\mathrm{R}$ be a ring for all $\mathrm{a} \in \mathrm{N}(\mathrm{R})$ and any I right or left ideal of $\mathrm{R}$ such that $\mathrm{aR} \subseteq \mathrm{I}$. Then the following condition are equivalent:

1 - $\mathrm{R}$ is right $\mathrm{n}$-weakly regular.

2- For all $\mathrm{a} \in \mathrm{N}(\mathrm{R}), \mathrm{aRI}=\mathrm{aR}$.

\section{Proof:}

$1 \rightarrow 2$ by Proposition 2.3.

$2 \rightarrow 1$ Let $\mathrm{I}=\mathrm{aR}$ and by Proposition 2.2.

\section{Proposition 2.5}

\section{Proof :}

Let $R$ be a right n-weakly regular ring. Then $N(R) \cap \operatorname{Cent}(R)=0$

If $N(R) \cap \operatorname{Cent}(R) \neq 0$, then there exists $0 \neq a \in N(R) \cap \operatorname{Cent}(R)$ such that $a^{2}=0$. since $\mathrm{R}$ is right $\mathrm{n}$-weakly regular then there exists $\mathrm{y}, \mathrm{z} \in R$ such that $\mathrm{a}=\mathrm{ayaz}=\mathrm{a}^{2} \mathrm{yz}=0 \mathrm{yz}=0$ $(\mathrm{a} \in \operatorname{Cent}(R))$. Therefore $a=0$. This is shows that $\quad N(R) \cap \operatorname{Cent}(R)=0$.

\section{Corollary 2.6}

Let $\mathrm{R}$ be a commutative ring. Then $\mathrm{R}$ is reduced if and only if $\mathrm{R}$ is right $\mathrm{n}$ weakly regular.

\section{Lemma $2.7[2]$}

1- Every one sided or two sided nil ideal of $R$ is contained in $J(R)$.

2- In any ring $R, a \in J(R)$ if and only if 1 -ar is invertible for all $r \in R$.

Now we have the following proposition

\section{Theorem 2.8}

Proof:

Let $R$ be a right $n$-weakly regular ring. Then $N(R) \cap J(R)=0$.

If $\mathrm{a} \in \mathrm{N}(\mathrm{R}) \cap \mathrm{J}(\mathrm{R})$, then there exists $\mathrm{y}, \mathrm{z} \in \mathrm{R}$ such that $\mathrm{a}=$ ayaz. Hence $\mathrm{a}(1-\mathrm{yaz})=0$, since $\mathrm{a} \in \mathrm{J}$, yaz $\in \mathrm{J}$, then by Lemma 2.7(2), there exists invertible element $\mathrm{v} \in \mathrm{R}$ such that $(1-y a z) v=1$. So $(a-a y a z) v=a$, yield $a=0$. Therefore $\quad N(R) \cap J(R)=0$.

Let $\mathrm{R}$ be a ring we denoted to the upper nil radical for a ring $\mathrm{R}$ by $\mathrm{Nil}^{*}(\mathrm{R})$ and it is the sum of nil ideal in the ring $\mathrm{R}$.

\section{Corollary 2.9}

\section{Proof:}

Let $\mathrm{R}$ be a right $\mathrm{n}$-weakly regular ring .Then $\mathrm{Nil}^{*}(\mathrm{R})=0$.

Let I be a left or right or two sided nil ideal, by Lemma 2.7(1), we have that $\mathrm{I} \subseteq \mathrm{J}(\mathrm{R}), \mathrm{I} \subseteq \mathrm{N}(\mathrm{R}) \cap \mathrm{J}(\mathrm{R})=0$, Theorem $2.8, \mathrm{I}=0$, which is a contradiction. So $\mathrm{R}$ not contain any nil ideal. Therefore $\mathrm{N}^{*}(\mathrm{R})=0$.

\section{Corollary 2.10}

Proof:

Let $\mathrm{R}$ be a right $\mathrm{n}$-weakly regular ring. Then $\mathrm{R}$ is semiprime ring.

Let $\mathrm{I}$ be a nilpotent right ideal then $\mathrm{I} \subseteq \mathrm{N}(\mathrm{R}) \cap \mathrm{J}(\mathrm{R})=0$ (Theorem 2.8) $\mathrm{I}=0$. Therefore $\mathrm{R}$ is semiprime ring.

\section{The Connection between n-Weakly Regular Rings and Other Rings.}


In this section we gives the connection between n-weakly regular rings and reduced rings, SNF rings.

\section{Proposition 3.1}

The following conditions are equivalent for a ring $\mathrm{R}$.

$1-\mathrm{R}$ is reduced.

2- $R$ is right $n$-weakly regular and $N(R)$ forms a right ideal of $R$.

3- $R$ is right n-weakly regular and $N(R)$ forms a left ideal of $R$.

4- $\mathrm{R}$ is right $\mathrm{n}$-weakly regular and NI ring.

5- $R$ is right n-weakly regular and $N(R) \subseteq J(R)$.

\section{Proof:}

$1 \rightarrow 4 \rightarrow 3 \rightarrow 5,1 \rightarrow 2 \rightarrow 5$ it is trivial.

$5 \rightarrow 1$

Suppose that $R$ is right $n$-weakly regular ring. So $N(R) \cap J(R)=0$, (Theorem 2.8). Since $N(R) \subseteq J(R)$, then $N(R) \cap J(R)=N(R)=0$. Therefore $R$ is reduced.

\section{Theorem 3.2} equivalent:

Let $\mathrm{R}$ be a ring with $\mathrm{aR}=\mathrm{Ra}$, for all $\mathrm{a} \in \mathrm{N}(\mathrm{R})$. Then the following conditions are

1 - $\mathrm{R}$ is right $\mathrm{n}$-weakly regular.

2- $\mathrm{R}$ is n-regular.

3- $\mathrm{R}$ is reduced.

\section{Proof:}

$1 \rightarrow 2$

Let $0 \neq a \in R$, such that $a^{2}=0$. Since $R$ is a right $n$-weakly regular, then $\mathrm{a} \in \mathrm{aR}=\mathrm{aRaR}$ (Proposition 2.1)

$=\mathrm{aRRa}(\mathrm{Ra}=\mathrm{aR})$

$=\mathrm{aRa}$.

so $\mathrm{a} \in \mathrm{aRa}$, hence $\mathrm{R}$ is $\mathrm{n}$-regular.

$2 \rightarrow 3$

Let $0 \neq a \in R$, such that $a^{2}=0$. Since $R$ is a right $n$-regular, then there exists $b \in R$ such that $a=a b a$ since $a R=R a$ there exists $x \in R$ such that $a b=x a$, so $a=a b a=x a^{2}=x 0=0$. Therefore $\mathrm{R}$ is reduced.

$3 \rightarrow 1$ It is trivial.

A ring $\mathrm{R}$ is called NCI provided that $\mathrm{N}(\mathrm{R})$ contains a non zero ideal of $\mathrm{R}$ whenever $\mathrm{N}(\mathrm{R}) \neq 0[1]$.

\section{Lemma 3.3 [1]}

Let $\mathrm{R}$ be a ring with $\mathrm{N}(\mathrm{R}) \neq 0$. Then $\mathrm{R}$ is $\mathrm{NCI}$ if and only if $\mathrm{N}^{*}(\mathrm{R}) \neq 0$.

\section{Proportion 3.4}

\section{Proof:}

Let $\mathrm{R}$ be a NCI ring, then $\mathrm{R}$ is right $\mathrm{n}$-weakly regular if and only if $\mathrm{R}$ is reduced.

Let $N(R) \neq 0$, sinc $R$ is NCI ring from Lemma 3.3, we get that $N^{*}(R) \neq 0$ but $R$ is right $n$-weakly regular, $N^{*}(R)=0$ (Corollary 2.9 ), which is contradiction. So $N(R)=0$. Therefore $\mathrm{R}$ is reduced.

A ring $\mathrm{R}$ is called weakly reversible if and only if for all $\mathrm{a}, \mathrm{b}, \mathrm{r} \in \mathrm{R}$ such that $\mathrm{ab}=0$, Rbra is a nil left ideal of $\mathrm{R}$ (equivalently braR is nil right ideal of R). Clearly ZI ring is weakly reversible [4]. 


\section{Proposition 3.5} reduced.

A ring $\mathrm{R}$ be right $\mathrm{n}$-weakly regular ring and weakly reversible if and only if $\mathrm{R}$ is Proof:

Let $a \in R$ with $a^{2}=0$. Then $a=a y a z$ for some $y, z \in R$ because $R$ is right $n$ weakly regular ring. Since $\mathrm{R}$ is weakly reversible then ayaR is nil right ideal of $\mathrm{R}$ so ayaR $\subseteq J(R) \cap N(R)=0$ (Lemma 2.7(1) \& Theorem 2.8) we get ayaR=0, in particular $\mathrm{a}=\mathrm{ayaz}=0$. Therefore $\mathrm{R}$ is reduced.

Converse, it is trivial.

Recall that a ring $\mathrm{R}$ is right $M C 2$ if $K \cong e R$ is simple, $\mathrm{e}^{2}=\mathrm{e}$, , then $\mathrm{K}=\mathrm{gR}$ for some $\mathrm{g}^{2}=\mathrm{g}[5]$.

\section{Lemma 3.6 [9]}

Let $\mathrm{R}$ be a left MC2,right SNF ring and MELT ring. Then $\mathrm{R}$ is a semiprime ring and right non singular.

\section{Theorem 3.7 [9]}

Let $\mathrm{I}$ be a right ideal of a ring $\mathrm{R}$. Then $\mathrm{R} / \mathrm{I}$ is $\mathrm{N}$ flat if and only if $\mathrm{Ia}=\mathrm{I} \cap \mathrm{Ma}$ for all $a \in N(R)$.

\section{Theorem 3.8} ring.

Let $\mathrm{R}$ be right SNF, left MC2 and MELT ring. Then $\mathrm{R}$ is left $\mathrm{n}$-weakly regular

\section{Proof:}

From Lemma 3.6, we get that $R$ is a semiprime ring and $a \in N(R)$. If $R a R+l(a)$ $\neq \mathrm{R}$, then there exists a maximal left ideal $\mathrm{M}$ of $\mathrm{R}$ containing $\mathrm{RaR}+\mathrm{l}(\mathrm{a})$, if $\mathrm{M}$ is not essential then we can write $M=l(e)$, where $e^{2}=e \in R$ and $e \neq 0$, since $R a R e=0$ because $\mathrm{RaR} \subseteq \mathrm{M},(\mathrm{ReRa})^{2}=0$ implies $\mathrm{ReRa}=0$ (since $\mathrm{R}$ is semiprime) $\mathrm{ReRa}=0$ in particular ea $=0$ and $\mathrm{e} \in \mathrm{l}(\mathrm{a}) \subseteq \mathrm{M}=\mathrm{l}(\mathrm{e})$, then $\mathrm{e}^{2}=0$, which is a contradiction. Therefore $\mathrm{M}$ is an essential, since $\mathrm{R}$ is MELT ring, then $\mathrm{M}$ is a two sided ideal then there exists a maximal right ideal $\mathrm{L}$ in $\mathrm{R}$ containing $\mathrm{M}$, since $\mathrm{R}$ is right $\mathrm{SNF}$ ring then $\mathrm{R} / \mathrm{L}$ is $\mathrm{N}$ flat right $\mathrm{R}$-module, $\mathrm{a}=\mathrm{ma}$ for some $\mathrm{m} \in \mathrm{M}$ (Theorem 3.7), (1-m)a=0, $1-\mathrm{m} \in \mathrm{l}(\mathrm{a}) \subseteq \mathrm{M} \subseteq \mathrm{L}$ therefore $1-\mathrm{m} \in \mathrm{L}$ implies $1 \in \mathrm{L}$ which is a contradiction, therefore $R a R+1(a)=R$ for all $a \in N(R)$. Thus $R$ is left n-weakly regular ring.

\section{Theorem 3.9}

Let $R$ be MELT and right SNF ring with RaR is essential for all $a \in N(R)$, Then $\mathrm{R}$ is left n-weakly regular ring.

\section{Proof:}

Let $a \in N(R)$. If $R a R+l(a) \neq R$ then there exists a maximal left ideal $M$ of $R$ containing $R a R+1(a)$, since $R a R$ is left annihilator of a nilpotent element by the hypothesis $\mathrm{RaR}$ is essential left ideal in $\mathrm{R}, \mathrm{M}$ is an essential ideal of $\mathrm{R}$ (MELT ring), there exists a maximal right ideal $L$ in $R$ such that $M \subseteq L$. Since $R$ is right $S N F$ ring, then $R / L$ is $N$ flat right $R$-module, $a=m a$ for some $m \in M$ (Theorem 3.7), $1-m \in 1(a)$ $\subseteq \mathrm{M} \subseteq \mathrm{L}$ implies $1 \in \mathrm{L}$, which is a contradiction. Therefore $\mathrm{RaR}+\mathrm{l}(\mathrm{a})=\mathrm{R}$ for all $a \in N(R)$, and so $R$ is left n-weakly regular ring.

\section{Definition 3.10 [8]}


A right $R$-module $M$ is said to be nil-injective, if for any $a \in N(R)$, any right $R$ homomorphism $f: a R \rightarrow M$ can be extended to $R \rightarrow M$, or equivalently $f=m$., where $m \in M$.

The ring $\mathrm{R}$ is called right nil-injective if $R_{R}$ is right nil-injective. Clearly a reduced ring is a right nil-injective and n-regular ring is a right nil-injective [8].

\section{Theorem 3.11}

Let $\mathrm{R}$ be a semiprime ring whose simple singular right $\mathrm{R}$-module are nilinjective. Then $\mathrm{R}$ is right $\mathrm{n}$-weakly regular ring.

\section{Proof:}

Let $a \in N(R)$. We claim that $R a R+r(a)=R$ if not, there exists a maximal right ideal $M$ of $R$ containing $R a R+r(a)$. If $M$ is not essential in $R$ then $M=r(e), e^{2}=e \in R$. Since $R a e \subseteq R a R \subseteq M=r(e)$, eRae $=0,(a e R)^{2}=0$ but $R$ is semiprime, aeR $=0$, so ae $=0$. Thus $\mathrm{e} \in \mathrm{r}(\mathrm{a}) \subseteq \mathrm{M}=\mathrm{r}(\mathrm{e})$, which is a contradiction. Hence $\mathrm{M}$ is essential right ideal in $\mathrm{R}$ and so $\mathrm{R} / \mathrm{M}$ is nil-injective. Define a mapping $f: a R \rightarrow R / M$ such that $\mathrm{f}(\operatorname{ar})=\mathrm{r}+\mathrm{M}$, let $\mathrm{x}, \mathrm{y} \in \mathrm{R}$ such that $a x=a y, a(x-y)=0, \quad(x-y)+M=f(a(x-y))=f(0)=M$, then $x-y \in M, \quad x+M=y+M$, $\mathrm{f}(\mathrm{ax})=\mathrm{x}+\mathrm{M}=\mathrm{y}+\mathrm{M}=\mathrm{f}($ ay $), \mathrm{f}$ is well define. $1+\mathrm{M}=\mathrm{f}(\mathrm{a})=(\mathrm{b}+\mathrm{M})(\mathrm{a}+\mathrm{M})=\mathrm{ba}+\mathrm{M}, \quad 1-b a \in \mathrm{M}$, since $b a \in R a R \subseteq M$ then $1 \in M$ which is a contradiction, so $R a R+r(a)=R$, in particular there is $y, z \in R$ and $v \in r(a)$ such that $y a z+v=1$, ayaz $+a v=a$, $a=a y a z$. Therefore $R$ is right n-weakly regular ring.

\section{Proposition 3.12}

Let $\mathrm{R}$ be a ring whose simple right $\mathrm{R}$-module are nil-injective. Then $\mathrm{R}$ is right $\mathrm{n}$ weakly regular ring.

\section{Proof:}

Assume that $a \in R$ such that $a R a=0$. Then $R a R \subseteq r(a)$. If $a \neq 0$ then there exists $a$ maximal right ideal $\mathrm{M}$ containing $\mathrm{r}(\mathrm{a})$. By hypothesis $\mathrm{R} / \mathrm{M}$ is nil-injective. We define a mapping $f: a R \rightarrow R / M$ such that $\mathrm{f}(\operatorname{ar})=\mathrm{r}+\mathrm{M}$, $\mathrm{f}$ is well define similar to Theorem 3.11, so there exists $b \in R$ such that $1+M=f(a)=b a+M, 1-b a \in M$ because $b a \in R a R \subseteq M$ then $1 \in \mathrm{M}$ which is a contradiction, so $\mathrm{a}=0$. Therefore $\mathrm{R}$ is a semiprime ring, by Theorem 3.11 we get that $\mathrm{R}$ is right $\mathrm{n}$-weakly regular ring. 


\section{REFERENCES}

[1] Hwang, S. U., Jeon, Y. Ch. and Park, K. S. (2007); “On NCI rings”, Bull. Korean Math. Soc., Vol. 44, No. 2, pp. 215-223.

[2] Kasch, F. (1982) "Modules and Rings" Academic Press Inc. (Londeon) Ltd.

[3] Kim, N. K., Nam, S. B. and Kim, J. K. (1999); “On simple singular GP-injective modules" Comm. In Algebra, Vol. 27, No.5., pp. 2087-2096.

[4] Liang, Z. and Gang, Y. (2007); “On weakly reversible rings” Acta Math. Univ. Comentanae, Vol. LXXVI, No. 2, pp. 189-192.

[5] Nicholson, W.K. and Yousif, M. F. (1997) "Mininjective ring" Journal of algebra, Vol. 187, pp. $548-578$.

[6] Ramamurthi, V. S. (1973) "Weakly regular ring" Canda. Math . Bull., Vol. 16, No. 3, pp.

[7] Stenström, B. (1977); "Ring of Quotient" Springer-Verlag, Berlin Heidelberg, New York.

[8] Wei, J. and Chen, J. (2007); "Nil-injective rings", International Electronic Journal of Algebra, Vol. 2, No., pp. 1-21.

[9] Wei, J. and Chen, J. (2008); "NPP rings, reduced rings and SNF rings", International Electronic Journal of Algebra, Vol. 4, No. , pp. 9-26.

[10] Yue Chi Ming, R. (1980) "On V-rings and prime rings” Journal of algebra, Vol. 62, pp. 13-20.

[11] Yue Chi Ming, R. (1983) "On quasi-injectivity and Von Neumann regularity" Mh. Math., Vol. 95, pp. 25-32. 